\title{
POST TRAUMATIC STRESS DISORDER: AN ILLNESS OF RECOVERY
}

\author{
Sengar K S \& Singh Archana
}

\begin{abstract}
Post traumatic Stress Disorder are the disorders caused in reaction to occurrence of some unacceptable, unpleasant incidence leading to intolerable stress/anxiety. It may be natural or manmade like war, disaster, rape, torture etc. These disorders are found $1-3$ percent in general population. All most seventy percent people of the general population experiences PTSD any time in their life time. Persistent avoidance of stimuli causing trauma is main feature of this entity. It can occur any time in life but most common in young adults. Most people do not experience the post traumatic catastrophic symptoms after passing the traumatic phase or some time after trauma the recovery starts taking place. However, significant number of people continuously experiences such symptoms and needs specialized intervention. The personality of the individual also plays a vital role on experiencing trauma and its recovery.
\end{abstract}

Post traumatic stress disorder (PTSD) is a condition marked by the development of symptoms after exposure to traumatic life events. The person reacts to this experience with fear and helplessness, persistently relives them, and tries to avoid being reminded of it.

\section{HISTORICAL BACKGROUND-}

18th Century: During Napoleon's time the Diagnosis of Nostalgia was established to describe Combat Stress Reaction. "Soldier's heart" was the name given during the US Civil War to a syndrome similar to PTSD. In 1866 the English Surgeon Erickson in his article he attributed conspicuous psychological abnormalities caused due to railway accidents to micro-traumas of the spinal cord, which later on led to the concept of "Railway Spine Syndrome". Jacob Da costa's (1871). in his paper, "On Irritable Heart," described soldiers with this syndrome. "Trauma Etiology" given by Charcot and Janet, pointed out the importance of traumatic experience for origin of hysterical symptoms. During the U S Civil War and World War I, same symptoms were referred to as the 'Da costa Syndrome' was noted among soldiers during the time of stress, especially when fear was involved. World War I (1914- 1918):: Charles Myres, British Psychiatrist introduced the term "Shell Shock" caused by direct exposure to shelling or bombing. World War-II veterans, survivors of Nazi concentration camps, and survivors of the atomic bombings in Japan had similar symptoms, was called combat neurosis or operational fatigue. The psychiatric morbidity associated with Vietnam War veterans finally brought the concept of PTSD. During
World War II (1939-1945). PTSD was known as New Syndrome as Combat Stress Reaction, Combat Neurosis/Battle Fatigue. PTSD as a formal Diagnosis was recognized in the year 1980 in DSM III with the emphasis that trauma could produce chronic symptoms in "normal" individuals too. Prior to that if symptoms were found persistent it was due to preexisting psychological problem. The official recognition of posttraumatic stress disorder (PTSD). in the DSM-III (American Psychiatric Association, 1980). has prompted considerable body of research into the psychology, biology, epidemiology, and also in the area of its treatment.

\section{Epidemiology of PTSD:}

The life time "incidence" of PTSD is estimated to be 9 to $15 \%$ and life time "prevalence" of PTSD is estimated to be about 8 percent of the general population. Among high risk groups whose members experienced traumatic events, the lifetime prevalence rates ranges from 5 to $75 \%$. The life time prevalence ranges from about 10 to $12 \%$ among women and 5 to $6 \%$ among man. Although PTSD may appear at any age but it is most prevalent in young adults, because they tend be more exposed to precipitating situations. (DSM-IV). 


\section{Co-morbidities:}

Although, the diagnostic features of posttraumatic stress disorder (PTSD) are well defined but the condition is not always easy to recognize. Studies in primary care settings shows, recognition rates as low as $2 \%$ have been reported. PTSD is often comorbid with obsessive-compulsive disorder, phobic disorder, panic disorder, generalized anxiety disorder, and depression. Other comorbid conditions found in association with PTSD includes bipolar disorder, somatization disorder, substance abuse or dependence, and eating disorders. PTSD is also associated with a high rate of attempted suicide (Jonathan, 2003). PTSD patients are 6 times more likely to attempt suicide than controls. PTSD results in more suicide attempts than in all other anxiety disorders. (Kessler, et al., 1999).

\section{The Current Diagnostic Criteria for PTSD are Unsatisfactory:}

It is difficult to distinguish between the PTSD symptom cluster and what could be considered a normal human response to massive trauma. PTSD, generalized anxiety disorder, depressive disorder, and substance dependence are difficult to separate. Symptoms that define PTSD are not strongly correlated with trauma. The diagnosis of PTSD relies on unverified trauma and subjective reports of symptoms. Financial compensation is an important reinforcing factor suggesting secondary gain. (Wilson \& Barglow, 2009).

\section{Clinical Features:}

PTSD is characterized by recurrent and intrusive recollections of the stressful event, either in images, thoughts, or perception or in dreams. There is an associated sense of re-experiencing of a stressful event and also marked avoidance of the events or situations that arouse recollections of the stressful event, feeling of numbness, and anhedonia. Reminders of trauma arouse intense distress and physiological reactions and are consequently avoidance, including conversation about the event. Patients try to push memories of the event out of their mind and avoid thinking about event in detail, particularly about it worst moments. On the other hand, many ruminate excessively about questions that prevent them from coming to terms with event, for example about why the event happened to them, how it could have been prevented, or how they can take revenge. The patient's emotional state ranges from intense fear, anger, sadness, guilt, or shame to emotional numbness. They often describe feeling detached from other people and give up previously significant activities. Various symptoms of hyperarousal include hypervigilance, exaggerated startle responses, irritability, difficulty concentrating, and sleep problems.

\section{Tools for Assessing PTSD:}

\section{The PTSD Checklist}

The PTSD checklist has two versions.

1. Military Version which was developed by Weathers F, Huska J, and Keane T, in year 1991.

2. Civilian Version, this scale was developed in year 1999 by Smith, M.Y, Redd, Duhamel et.al. Both scales contain 17 items. In military version items are related to the stressful military experiences and in civilian version items are a list of problems and complaints that people sometimes have in response to stressful experiences.

\section{Impact of Event Scale (IES):-}

Impact of Event Scale (IES) was developed by Horowitz, M., Wilner, M., and Alvarez,W. in year 1979. The IES is a self administered questionnaire evaluating experiences of avoidance and intrusion which attempts to. IES was developed to measure current subjective distress related to a specific event. The IES scale consist of 15 items, 7 of which measure instructive symptoms, and 8 tap avoidance symptoms. The combined scale provides a total subjective scale score. All items of the IES are anchored to a specific stressor. Both the intrusion and avoidance scales have displayed acceptable reliability (.79 and .82, respectively). The IES has also displayed the ability to discriminate a variety of traumatized groups from non traumatized groups. It is an instrument that can be used for repeated measurement over period of time. Its sensitivity to change situation is useful for monitoring the patient's progress in therapy. The Items can be interpreted according to following dimensions:

0-8 Subclinical Range

9-25 Mild Range

26-43 Moderate Range

44+ Severe Range.

\section{Secondary Traumatic Stress Scale (STSS).}

Secondary Traumatic Stress Scale (STSS). is a 17-items self-report instrument designed to measure intrusion, avoidance, and arousal symptoms associated with direct exposure to traumatic events via one's professional relationships with traumatized clients.

\section{The Clinician-Administered PTSD Scale (CAPS).}

The CAPS (Blake et al, 1995) is considered as the "gold standard" of structured interviews for post traumatic stress disorder. The CAPS has several helpful features, including a standard prompt questions and explicit 
behaviorally anchored rating scale and assess both frequency and intensity of symptoms. It generate both dichotomous and continues scores for current (one month). and life time ("worst ever"). PTSD. In addition to the standard 17 PTSD items, the CAPS also contains items tapping post traumatic impacts on social and occupational functioning, improvement in PTSD symptoms since a previous CAPS assessment, overall response validity and overall PTSD severity, as well as items addressing guilt and dissociation. CAPS may require an hour or longer to complete administration, may sometime provide more information than actually is needed clinically, and focuses only on PTSD

\section{Trauma Symptoms Checklist}

The TSC-40 by Elliot \& Briere (1992) is a research measure that evaluates symptomatology in adults associated with childhood or adult traumatic experiences. It measures various aspects of posttraumatic stress and other symptoms clusters found in some traumatized individuals. The TSC-40 is a 40-item self-report instrument consisting of six subscales: Anxiety, Depression, Dissociation, Sexual Abuse Trauma Index (STI)., Sexual Problems, and Sleep Disturbance, can be measured separately as well as a total. Each symptom item is rated according to its frequency of occurrence over the prior two months, using a four point scale ranging from 0 ("never") to 3 ("Often"). The TSC-40 requires approximately 10-15 minutes to complete and can be scored in 10-15 minutes.

\section{Theories-old and recent}

\section{Early theories can be divided into three groups}

- Social-cognitive theories primarily focus on the way trauma breaches existing mental structures and on innate mechanisms for reconciling incompatible information with previous beliefs. Social-cognitive theories provide good accounts of the range of emotions and beliefs occasioned by trauma and of the process of long-term adjustment, without clearly differentiating between PTSD and other types of reaction such as neither depression, nor do they account for the nature of responses to trauma reminders.

- Conditioning theories deal with learned associations and avoidance behavior. Conditioning theory provides a good account of how trauma cues acquire the ability to elicit fear and of the critical role played by avoidance, but is limited by the absence of cognitive elements in explaining many of the symptoms and data concerning PTSD, especially those dealing with beliefs and perceived threat.

- Information-Processing Theories focus on the encoding, storage, and recall of fear-inducing events and their associated stimuli and responses. Information-processing theories offer clearer descriptions of the cognitive architecture by which the traumatic event may be represented, of effects on attention, and of how the overturning of assumptions increases the number of potential trauma reminders, but are less able to account for the importance of emotions other than fear and of beliefs extending beyond issues of danger to the wider social context.

\section{Early theories:}

\section{Horowitz's Formulation of Stress Response Syndrome:}

The Horowitz (1973) formulation of stress response syndrome is the most influential cognitive model of reaction to trauma to date. Although derived from the classical psychodynamic psychology, Horowitz's theory is principally connected with discussing such ideas in terms of cognitive processing of traumatic formation such as ideas, thoughts, images, affects and so on. Horowitz has argued that the main inputs within the cognitive system for the processing of trauma related information comes from a completion tendency, the psychological need to match new information with inner models based on older information, and the revision of both until they agree."This tension causes the individual to oscillate between phases of intrusion and denial -numbing as they gradually integrate with long -term meaning representations. The failure of such processing can remain in active memory ever being fully assimilated, thus leading to chronic post traumatic reactions

\section{Janoff-Bulman's Cognitive Appraisal Theory:}

The cognitive-appraisal model of Janoff-Bulman (1985) centers almost exclusively on the content of the preexisting beliefs about the self and the world which the individual carries into a traumatic situation. JanoffBulman argues that PTSD is the result of certain basic assumptions about self and the world being "shattered". The assumption which Janoff-Bulman is referring to are the assumption of invulnerability, the perception of the world as a meaningful or comprehensible or and the view of the self in a positive light. The proposal is that these assumptions provide a structure and meaningful in the individuals life. But that they cannot be maintained in the phase of a traumatic experience and consequently they "shattered', plunging the individual into a confusion of intrusion, avoidance and hyper arousal 


\section{Foa's Fear Network:}

Applying Lang's theory of fear structures foa \& Kozak (1986) and her colleagues have outlined an information processing theory of PTSD which centers on the formation of a so-called fear network in long term memory. This fear net work encompasses a stimulus information about the traumatic event, information about cognitive, behavioral and physiological reactions to the event and information which links these stimulus and response elements together. Activation of the trauma related fear network by cue stimuli, according to Foa causes information in the network to inter conscious awareness (the intrusion symptoms of PTSD).

\section{Cognitive Action Theory:}

The cognitive action theory of Chemtob et al., (1988) is a product of research with veterans of war in Vietnam. According to this theory individuals with PTSD, the fear net work is permanently activated causing them to function in a 'survival mode" thus a way of functioning that was adaptive during the traumatic incident. This permanent activation of the network, it is suggested, leads to the symptoms of hyper arousal and intrusion. Such symptoms are exacerbated by to further functions of the Chemtob et al. model. First, the increase "gain" of the system in PTSD means that vicious circles of increasing arousal operate more quickly and secondly, it is suggested that PTSD sufferers have higher limits on the magnitude to which the threat - arousal system can be activated.

\section{Information-Processing Theory:}

The cognitive processing model of PTSD of Creamer et al, (1992) is a derivative approach presented as a "synthesis and reconceptualization of existing formulation". Creamer et al argue for an initial period of intrusion (due to activation of the fear network) with which the individual copes by the calling upon a range of defense and avoidant strategies. More over Creamer et al. suggest that this initial intrusive experience can be used as an index of the degree of net work resolution processing and is occurring. In this analysis, then, high levels of initial intrusion are a predictor of successful recovery whereas low level of initial intrusion is a predictor of poor outcome and chronic pathology.

\section{Recent Theories:}

\section{Brewin's Dual Representation Theory:}

Brewin's et al. (1995) endeavors to circumvent the short comings of single level theories by proposing two levels in memory at which trauma related information can be represented. The first level of representation is of the individual's conscious experience of the traumatic event. This form what Brewin called verbally accessible memories (VAMS).VAMS are characterized by their ability to be deliberately retrieved and progressively edited by the traumatized individual. VAMS representations, it is argued, as with foa's network, contains sensory, response and meaning information about the traumatic event. The second level of representation proposed by Brewin consists of situationally accessible memories (SAMS). SAMS contains information which cannot be deliberately accessed by the individual and is not available for progressive editing. Dual representation theory propose that VAM and SAM representations are encoded in parallel at the time of trauma and between then account them account for the range of PTSD phenomenology.

\section{The SPAARS Approach:}

Schematic, Propositional, Associative and Anological Representational Systems by Dalgiesh \& Power, (1995). The SPAARS approach deal with emotions. It is a multi representational model with four levels/formats of representation in which two routes to the generation of emotions are specified. SPAARS is a functional theory of emotion. Within SPAARS emotions are emotional tools which the cognitive system employs to resolve problems with active, valued goals. So, for example if a goal is threatened, this will be appraised within SPAARS and a fear module will be activated. The fear module is essentially a reconfiguration of the cognitive system to deal with the imminent threat and any possible future threats. Within SPAARS, then, emotions are adaptive processes which recognize the cognitive system in different ways to deal these changes in the internal or external environment. The suggestion is that the adaptiveness of emotions can sometime go awry and this can lead to the development and maintains of so called emotional disorders.

Ehlers and Clark's Cognitive Model provides what is currently the most detailed account of the maintenance and treatment of PTSD. They have significantly expanded understanding of the wide range of relevant negative appraisals and have identified both appraisals and a variety of cognitive coping factors that influence the course of the disorder. These aspects of the model have been strongly and consistently supported by empirical research. 


\section{Models of Psychopathology in PTSD}

\section{Anxiety disorder:}

- Fear, anxiety and avoidance behaviour (as with phobias).

- Intrusive phenomena resemble obsessive-compulsive disorder

- Introversion and neuroticism are common personality traits in both PTSD and anxiety

- $\quad$ Some people improve with exposure therapy

\section{Mood disorder:}

- Sadness and grief, independent of bereavement

- Co morbidity with depressionis very common

- Vegetative symptoms (loss of sleep, appetite, libido). are very similar

- Avoidance, numbing and loss of interest as in mood disorders

\section{Dissociative Disorder:}

- Flashbacks and amnesia are common

\section{Personality Disorder:}

- Considerable overlap of symptoms with borderline personality disorder

- Some overlap of symptoms with antisocial personality disorder (antisocial behaviour, irritability).

- Some evidence that trauma can induce personality change

\section{Separate Neurophysiological disorder:}

- Low monoamine oxidase activity

- Increased excretion of urinary beta-endorphin

- Therapeutic response to serotonergic drugs and drugs that affect the locus ceruleus

- Deregulation of hypothalamic-pituitary-adrenal axis resulting in low cortisol levels

\section{The Role of Persistent Dissociation in PTSD}

Briere and colleagues (2005). highlights the importance of persistent dissociation in the etiology of posttraumatic stress disorder (PTSD). On the basis of two crosssectional studies, the authors conclude that "the primary risk for PTSD is less whether one dissociates during (or soon after). a traumatic event than whether such dissociation persists over time". Murray (2002). reported two prospective longitudinal studies of motor vehicle accident survivors and came to a remarkably similar conclusion: "Persistent dissociation and rumination at 4 weeks after trauma are more useful in identifying those patients who are likely to develop chronic PTSD than initial reactions". Participants were assessed very soon after the accident and followed for 6 months. Persistent dissociation at 4 weeks was a better predictor of chronic PTSD at 6 months than peritraumatic dissociation measured in the immediate aftermath of the trauma. Halligan et al (2003) showed that persistent dissociation predicted an additional $8 \%$ variance of PTSD severity at 6 months over and above what could be predicted from trauma severity and cognitive processing measures, including peritraumatic dissociation.

\section{Risk Factors for PTSD}

- Women are twice as likely to develop PTSD

- Being 40 to 60 years old

- Being a member of an ethnic minority group

- Low SES

- History of emotional problems

- Prior history of trauma

- Living in a highly disrupted or traumatized community, loss of income or livelihood

- Having on-going psychosocial stressors

- Higher level of exposure, perceived threat to life

$>$ Aspect of Trauma

- Duration andmagnitude of exposure to stressor

- $\quad$ Stressors are sudden and/oroccur with no warning

- $\quad$ There is multiple loss of life, mutilation or grotesque imagery

- Criminal violence, especially sexual

$>$ Experienceduring Trauma

- Perceived own life to be at real risk

- $\quad$ Perceived lack of control of events, intense fear and helplessness

- Perception of grotesque imagery, especially of human man or children

- Witnessing or carrying out atrocities, e.g. murder, torture

- High levels of dissociative symptoms at the time of the event

\section{> Characteristics of the Individual}

- Previous psychiatric illness or neuroticism

- Previous exposure to trauma, especially childhood trauma 
- $\quad$ Previous copingstyle

- Denial of trauma and/or avoidance

- Femalegender

- Previousacute stress reaction

$>$ Post-trauma

- Denial of trauma by others or dismissal of experience

- Lack of social support

\section{The Role of Spirituality in Recovery}

Trauma often seems to precipitate a spiritual crisis, in which the survivors struggle to establish a greater understanding of transpersonal issues which are often left unexplored within the traditional cognitive and cognitive - behavioral approach which are, by their nature, focused on the alleviation of so-called disorder and the symptoms. For many, the experience of trauma represents a powerful positive and existential change in outlook, the exploration and facilitation of which are not easily amenable to the techniques of cognitive and cognitive-behavioral therapies.

\section{Post-traumatic stress disorder (PTSD). and the family}

If a loved one has post-traumatic stress disorder (PTSD)., it's essential that you take care of yourself and get extra support. PTSD can take a heavy toll on the family if you let it. It can be hard to understand why your loved one won't open up to you - why he or she is less affectionate and more volatile. The symptoms of PTSD can also result in job loss, substance abuse, and other stressful problems.

Letting your family member's PTSD dominate your life while ignoring your own needs is a surefire recipe for burnout. In order to take care of your loved one, you first need to take care of yourself. It's also helpful to learn all you can about post-traumatic stress disorder (PTSD). The more you know about the symptoms and treatment options, the better equipped you'll be to help your loved one and keep things in perspective.

\section{Issues To Be Taken Care of in Treatment}

- $\quad$ Areas of active and important debate on treatment of PTSD include the management of acute posttraumatic reactions and the prevention itself of PTSD after experience of traumatic events. For managing acute stress disorder, the literature supports the use of brief treatments that employ principles of cognitive restructuring and exposure to details of the event. This may include the prolonged exposure/cognitive restructuring described by others
(Bryant, 2003)., ornewer treatments such as memory structuring intervention (Gidron, 2001). Both of these treatments are administered in the course of two to four sessions in the immediate post trauma period, and they appear to lower the risk of subsequent PTSD.

A widely promoted intervention for preventing PTSD is psychological debriefing or critical incident stress debriefing. This technique, originally developed for use with rescue personnel, has been widely adopted but with insufficient critical examination. Recentassessments have demonstrated a lack of benefit for single-session debriefing as a means of preventing PTSD is of greater concern, however, is the possibility that such interventions actually worsen the prognosis. (Mayou et al., 2000).?

- Foa (2000). reviewed empirically validated psychosocial treatments for posttraumatic stress disorder (PTSD). and factors associated with successful therapy outcome. Most of the treatments whose efficacy was studied empirically fall within the broad category of cognitive-behavioral therapy. These include exposure therapy, anxiety management programs and cognitive therapy. These modalities of therapy have been developed to modify conditioned fear and erroneous cognitions that are thought to underlie PTSD. Exposure therapy has the most empirical support because it was found to be effective across different populations of trauma victims with PTSD. Combinations of therapies have also been used, and the value of these is discussed. In addition, recent evidence shows the efficacy of eye movement and desensitization reprocessing. A growing body of evidence supports the use of psychosocial treatments for PTSD, but not all patients benefit. Future research should develop programs that increase the motivation of patients to take advantage of these efficacious treatments.

- $\quad$ Randomized controlled trials have now produced positive results for multiple-session trauma-focused cognitive behavior therapy for survivors with acute stress disorder within a month of the trauma (Bryant et al. ,1999)., those with distressing traumatic stress symptoms 1 month after the trauma (Bisson et al ., 2004) and those with acute PTSD between 1 and 3 months after the trauma (Ehlers et al., 2003). These results led the authors capitulate the guidelines delineated by U. K. National Institute for Health and Clinical Excellence in 2005 to recommend that 
trauma-focused cognitive behavior therapy be made available to all individuals with acute PTSD between 1 and 3 months after a traumatic event (National Collaborating Centre for Mental Health,2005).

\section{How community respond to traumatic period}

Community responses to traumatic period are in four different ways -

Heroic Phase: Communities Pull Together

During and immediately following a disaster, individuals and communities often respond supportively, altruistically, and heroically.

Honeymoon Phase: In this attention stays focused on the victims. The community adopts the four common modes $t$ combat stressors-

1) Intense community mobilization

2) Increased community consensus

3) People from outside the community come to help

4) Organizations adapt to help the community.

Disillusionment Phase: The survivors of any traumatic events are provided the different type of help from different sources. However, they learn to pass time leisurely. But once helpers leave the once the survievers left alone to face the reality the disillusionment phase occurs. Where persons find them difficult ot face the reality and cope with new situations. This issue deals with following points -

- Longest phase of recovery.

- Immediate response teams leave.

- Assistance and help weakens.

- Losses become a reality.

- Widespread discouragement.

- Scapegoating, resentment, disagreement.

- Unity fades.

\section{Recovery and Reconstruction: Finding a New Normal} Life.

- May not occur for a year or more post-disaster.

- Social and economic activities recover.

- Gradual return to normal routines.

- Completion of reconstruction and recovery efforts.

- Community tries to find a new normal life.

- Anniversary events.

- Difficulty in recovery decisions is compounded by poor planning and preparedness.
Certain patterns are required to build a new pattern of starting newer phase of life after trauma which needs to be considered for coming back on track.

\section{Seven Supportive Communications:}

1. Empathy: "How are you holding up?"

2. Normalization: "You are having a normal reaction to abnormal events and situations."

3. Recognition of efforts to cope: "Everyone copes in his or her own way."

4. Self-care: "Make sure you are doing things to keep yourself healthy."

5. Tolerance for change: "You will find a new normal after this is over."

6. Instilling hope; "You have made it through some tough times before, and you will make it through this, too."

7. Accepting help: "It is okay to take some help when you need it."

Most often past traumatic problems recover gradually by its own by passing the time but still it require psychosocial intervention for better faster and safer recovery from the trauma and sometimes it also needs to be treated by pharmacological agents.

INDICATED TREATMENTS FOR POSTTRAUMATIC DISORDERS:

\section{Acutestress responses}

- Debriefing

- Social supports

- Pharmacologicalsupports, e.g. hypnotics

- Information and advice to families

2. AcutePTSD:

- Exposure therapy may be first-line treatment if intrusive phenomena prominent

- Cognitive therapy

- Briefpsychodynamicpsychotherapy

- Antidepressants (especially where avoidance prominent).

\section{ChronicPTSD:}

- Exposure therapy if trauma never discussed

- Cognitive-behavioural approaches may still be effective (group or individual).

- Long-termpsychotherapy (group or individual).

- Antidepressants, lithium, carbamazepine (National Institute of Clinical Excellence, 2005). 


\section{COMMUNITY \& CULTURE:}

Community has its own relevance and greater significance in cases of traumatic events. Community and culture plays significant role in recovery and following points must be considered during intervention.

- Psychosocial Interventions should be fully integrated with the overall relief and rehabilitation activities right from the beginning following a disaster. Respect for local cultures needed in implementing psycho-social interventions. All programmes must be culturally sensitive and appropriate to the local community. Understanding of local culture helps to determine the appropriateness and feasibility of specific interventions.

- Strong belief in traditional healers may affect the choice of interventions in many ways. Attitudes toward intervention is also very important (e.g., preference for or dislike of medication).;

\section{Why Early Interventions In PTSD?}

- There is a dearth of naturalistic, prospective studies of the course of posttraumatic recovery, especially the course of adjustment to mass violence and traumatic loss.

- About $90 \%$ people recover after stress management training, but what happens to those who don't.

- $\quad$ There is much conjecture about what puts people at risk for chronic PTSD, but there are few well defined studies.

- Well trained professional are scare, evaluating efficient methods of delivering the key elements of early interventions is crucial

- There is scant descriptive, epidemiological or clinical research on the unique psychosocial needs and outcomes of individuals who suffer from the dual burden of losing a loved one through trauma while experiencing their own acute trauma.

- But there is a paradigm shift in the area of early intervention well before 9-11

- Early intervention is dominated by non - evidence based practices, poorly defined and anarchical notions about recovery from trauma and the risk for trauma -linked disorders and an apparent unresponsiveness to scientific inquiry.

\section{Delay in Treatments (Perceived barriers in seeking treatment).}

Don't trust,

Too embarrassing,

Harm my career,

Colleagues are less confident in me,

Leaders blame me,

Seen as weak (Hoge et al, NEJM, July 1, 2004).

- Stigma

- Low awareness of PTSD

- Professional help not sought (24.5\%).

\section{PROGNOSIS OF PTSD}

- Effects on social systems and support

- As in general psychiatric practice, it is important to consider the influence of the disorder on other areas of a person's life, in relation to its effect on the process of recovery and the prognosis. The symptoms of many of the post-traumatic disorders may be troublesome for families and employers, particularly in the first 6-12 months. Families, friends and employers are usually unfamiliar with the timescale of normal recovery. Most people do not realize that normal recovery may take 6 months, or longer if there are further stressors, and they might become impatient with survivors, thinking them difficult or weak. After experiencing trauma a person may be chronically irritable and withdrawn for weeks, in a way which is alien to them and their families. There is good evidence that marital stress and breakdown are increased after traumatic experiences. Work performance may similarly deteriorate because of the person's hyper vigilance, accompanying loss of concentration and irritability. However, people often find it impossible to discuss the reasons for this with their employers. Employers may not be sympathetic anyway, especially if the trauma occurred at work (and compensation is being claimed)., or where there is a work culture of denial of distress. Many major personal disasters are never reported in the press. During peacetime, and between major disasters, the principal cause of traumatic stress responses is crime, of which the impact on the victim is rarely reported unless it is fatal. (Kilpatrick et al, 1989). This applies to both men and women. A good example of this is the plight of the families of murder victims. The killer is often a member of the family and relatives must cope with multiple losses. 


\section{Case: 1}

"Ms A. 45 years married with three children, presented with multiple problems after her husband murdered their daughter to prevent her from telling the mother about his 20-year affair with a family friend. This woman lost not only her daughter and her husband; she also lost experience of her marriage and the support of a trusted friend with whom she was confident that she/he will help at each movement. She was also without funds as the husband had been the principal earner and she did not have access to the bank account. The court trial did not take place for a year, and the funeral of the daughter was delayed several times due to some legal proceedings related to post-mortem reports for both defense and prosecution".

- These social and legal aspects of post-traumatic dysfunction have a profound influence on the management and prognosis of PTSD, and can cause major setbacks in treatment.

- A man who becomes homeless because of domestic violence related to his post-traumatic irritability may be unable to cooperate with or tolerate a treatment programme

- In-patient treatment might be indicated in such instances. People with PTSD as a result of crime have particular problems: not only they have reminders of the stressor, such as police identification parades or court appearances; they might be at continued risk of further trauma, such as threats from the defendant.

\section{Choosing a treatment}

There are particular questions relevant to the selection of treatment.

\section{What is the worst problem at the moment?}

If intrusive phenomena are prominent, this may suggest exposure therapy as part of a cognitive-behavioral package. If depression and distress are worst, then regular supportive therapy sessions plus antidepressants may be most effective. The support system must be assessed. What support does this person have and what solutions he is adopting?

\section{What supports does this person have?}

Many forms of treatment for PTSD are quite stressful. It is therefore important to ensure that the patient will be well supported, and that the families are informed about the natureand process of therapy.

\section{What solutions to stress are the patient adopting now?}

If a patient is misusing alcohol or drugs as a means of managing their PTSD symptoms this needs to be addressed before any specific PTSD treatment can be implemented. Rarely, patients present with acts of selfharm such as overdoses, and these should not be dismissed as 'attention-seeking'.

\section{Helping a loved one with PTSD}

- Be patient and understanding. Getting better takes time, even when a person is committed to treatment for PTSD. Be patient with the pace of recovery and offer a sympathetic ear. A person with PTSD may need to talk about the traumatic event over and over again. This is part of the healing process, so avoid the temptation to tell your loved one to stop rehashing the past and move on. Also try to anticipate and prepare for PTSD triggers. Common triggers include anniversary dates; people or places associated with the trauma; and certain sights, sounds, or smells. If you are aware of what triggers may cause an upsetting reaction, you'll be in a better position to offer your support and help your loved one to calm down.

- Don't take the symptoms of PTSD personally. Common symptoms of post-traumatic stress disorder (PTSD) include emotional numbness, anger, and withdrawal. If your loved one seems distant, irritable, or closed off, remember that this may not have anything to do with you or your relationship.

- Don't pressure your loved one into talking. It is very difficult for people with PTSD to talk about their traumatic experiences. For some, it can even make things worse. Never try to force your loved one to open up. Let the person know, however, that you're there when and if he or she wants to talk.

\section{INTEGRATING PSYCHOSOCIAL TREATMENT FOR PTSD \& SEVERE MENTAL ILLNESS:}

Patients with severe mental illness (SMI)., such as schizophrenia, bipolar disorder, and major depression, are also more likely to have experienced trauma in childhood, adolescence, and throughout their adult lives than the general population. (Bebbington et al, 2004, Goodman,1997). This high exposure to traumatic events such as physical and sexual abuse and assault takes a heavy toll. In addition to the immediate effects of victimization on an individual's quality of life, a history of trauma exposure in persons with SMI is associated with 
more severe symptoms, greater impairment of • functioning, and higher levels of distress (Briere J, 1997). During the past decade, research has firmly documented that one of the most common consequences of trauma in patients with SMI is their high vulnerability to posttraumatic stress disorder (PTSD). The lifetime prevalence of PTSD in the general population is $8 \%$ to 12\% (Breslau et al., 2004; Kessler et al, 2005) in comparison to patients with SMI have much higher rates of PTSD, with most reported estimates ranging from $29 \%$ to $47 \%$. (Calhoun et al, 2007; Mc Farlane et al, 2001; Mueser et al., 1998, Switzer et al, 1999). Two approaches have predominated in developing programs for SMI. First, some interventions focus specifically on PTSD by adapting treatment approaches shown to be effective in the general population for patients with SMI, such as cognitive restructuring (Mueser et al , 2004; Mueser et al 2007) and exposure therapy.(Frueh et al, 2004). Second, some interventions are more broad-based and address a wide range of trauma squeal, such as poor self-esteem and body image, dysregulated behavior, and problematic relationships. Pharmacological treatments are often included as important components of treatment. Research on the effects of programs specifically developed to address trauma in patients with SMI is still in its infancy. However, clinical reports from early trials of these programs indicate that patients with SMI can be successfully engaged and treated, and that outcomes often improve (Harris, 1998). (Rosenberg 2004). Only 1 randomized controlled trial of a treatment for trauma in SMI has been completed (K. T. Mueser et al, unpublished data, 2007). The results of the trial indicate that participation in the 12- to 16-week cognitive-behavioral therapy program was associated with improvements in PTSD symptoms, other symptoms such as depression, and trauma-related beliefs about oneself and the world

\section{CASE VIGNETTE}

- $\quad$ Elizabeth is a 46-year-old woman with schizophrenia and PTSD. As a child she experienced significant physical abuse from her mother and, in an attempt to escape the abuse, frequently ran away from home. As a result, she spent much of her adolescence in a children's home. At 17, she left the home and moved in with her stepfather, who was separated from her mother. She lived with him for about a year, during her stay with step father, he sexually abused her. At one point she became pregnant by him; she told no one, but suffered a painful and confusing miscarriage. Shortly thereafter, she withdrew from people and stopped taking care of herself, which was followed by signs of psychosis, including paranoia, hallucinations, and conceptual disorganization.
Elizabeth was hospitalized, treated, and discharged free of psychotic symptoms. She began to work as a store clerk, married, and had 2 children. Although her husband physically abused her, she remained fairly stable until he sought a divorce and took custody of their children. Following this, Elizabeth's condition worsened, and she spent several years cycling in and out of hospitals, interspersed with periods of homelessness.

- During a stay at a halfway house, she met a man and fell in love. Despite his drug abuse, they forged a relationship that lasted several years, during which time Elizabeth's symptoms and functioning stabilized, and she began working part-time. Then one day she found him dead, which was followed by deterioration in her functioning.

- Several years later, an evaluation confirmed that Elizabeth had severe PTSD. She reported that the death of her boyfriend was her most distressing traumatic experience. She said that she felt extremely guilty because she had not been able to prevent his death. She also talked about how her history of abuse made it difficult for her to trust anyone.

- $\quad$ The first session focused on teaching breathing retraining to manage her anxiety. During a homework review the following week, Elizabeth stated that she only felt comfortable practicing breathing retraining with her eyes open, but that she found it very helpful.

- $\quad$ The next 2 sessions focused on discussing the symptoms of PTSD, including how they differ from the symptoms of schizophrenia, and how they are affected by each other.

- Elizabeth expressed relief in learning that her PTSD symptoms were common reactions to traumatic experiences.

- During the fourth session cognitive restructuring was introduced. Elizabeth was able to understand the connection between thoughts and feelings but struggled with the related homework. She noted that she was afraid of becoming too overwhelmed when trying to do her homework alone.

- After more sessions in which the therapist helped her using cognitive restructuring to examine and challenge thoughts related to her upsetting feelings, Elizabeth began to practice using the skill on her own to deal with negative feelings.

- $\quad$ She described the therapy program as helpful and agreed to a joint session with her case manager at the end of treatment so that she and her therapist could teach her case management skills that Elizabeth had developed in therapy.

- A recurring concern that was addressed with cognitive restructuring was Elizabeth's fear that nobody would claim her body at her death. 
- She felt abandoned by her children, depressed and isolated, and preoccupied by worry about rejection. She was eventually able to identify several core beliefs, including the thoughts that "you cannot trust anybody" and "anyone that you do trust will abandon or mistreat you." Over the course of treatment she was able to recognize that there were some people who had stood by her (eg, her therapist, her psychiatrist, her case manager, her son).

$\varepsilon \quad$ She was able to gain a more balanced perspective on her feelings of guilt over her boyfriend's death, realizing she was not to blame. As Elizabeth's symptoms gradually improved, she began to reach out to others. She started having brief conversations with people (e.g, at the library)., reconnected with a neighbor, and renewed her relationship with her son.

- $\quad$ She felt abandoned by her children, depressed and isolated, and preoccupied by worry about rejection. She was eventually able to identify several core beliefs, including the thoughts that "you cannot trust anybody" and "anyone that you do trust will abandon or mistreat you." Over the course of treatment she was able to recognize that there were some people who had stood by her (eg, her therapist, her psychiatrist, her case manager, her son).

- She was able to gain a more balanced perspective on her feelings of guilt over her boyfriend's death, realizing she was not to blame. As Elizabeth's symptoms gradually improved, she began to reach out to others. She started having brief conversations with people (eg, at the library)., reconnected with a neighbor, and renewed her relationship with her son.

\section{Few important Issue on PTSD -}

- Early intervention is a must.

- Help to be seek from specially trained professionals.

- Increasing public awareness about trauma related disorders.

- Localization of the treatment programs.

- Client oriented individual therapies.

- Involvement of clients' social networks.

- $\quad$ Local leaders need to be included.

- IES materials need to be distributed.

- Evidenced based research.

- Outcome studies specific to different group of population.

Yet man is born unto trouble, as the sparks fly upward Job 5:7

\section{References:}

1. Acharya, Vedamurthy .,Descilo, Teresa.,\& Damodoran, R. (2005). Yoga Breath Program and Client-Centered Exposure for Relief of PTSD in Tsunami Victims. NIMHANS (UNDER PUBLICATION).

2. American Psychiatry Association (1994). Diagnostic and Statistical manual of mental disorders ( $4^{\text {th }} e d$. ). American Psychiatric Associatin, Washinton, DC.

3. Bebbington P E., Bhugra D., \& Brugha T, et al. (2004). Psychosis, victimization and childhood disadvantage: evidence from the second British National Survey of Psychiatric Morbidity. British Journal of Psychiatry, 185, 220-226.

4. Bisson J I, Shepherd J P, Joy D, Probert R, \& Newcombe RG (2004). Early cognitive-behavioural therapy for posttraumatic stress disorder symptoms after physical injury: randomized controlled trial. British Journal of Psychiatry, $184,63-69$

5. Breslau N, Peterson E L., \& Poisson L M, (2004). Estimating post-traumatic stress disorder in the community: lifetime perspective and impact of typical traumatic events. Psychological Medicine, 34, 889-898.

6. Brewin, C. R., Dalgleish, T., \& Joseph, S. (1995). A dual representation theory of post traumatic stress disorder. Manuscript submitted for publication.

7. Bride, Robinson, Yegidis, \& Figley. (2004). Development and Validation of the Secondary Traumatic Stress Scale. Research on Social Work Practice, 14, (1). 27-35

8. Briere, J., Scott C., \& Weathers F (2005). Peritraumatic and persistent dissociation in the presumed etiology of PTSD. American Journal of Psychiatry, 162, 2295-2301

9. Briere J., Woo R, \& McRae B., (1997). Lifetime victimization history, demographics, and clinical status in female psychiatric emergency room patients. Journal of Nervous and Mental Diseases, 185, 95-101.

10. Briere, J., \& Runtz, M. (1989). The Trauma Symptom Checklist (TSC-33). Early data on a new scale. Journal of Interpersonal Violence, 4, 151-163.

11. Bryant RA., Moulds M L., \& Nixon RV.(2003). Cognitive behaviour therapy of acute stress disorder: a four-year follow-up. Behaviour Research Therapy,41, 89-494

12. Bryant R A., Sackville T., Dang ST., Moulds M., \& Guthrie R (1999). Treating acute stress disorder: An evaluation of cognitive behavior therapy and supportive counseling techniques. American Journal of Psychiatry $156,1780-1786$

13. Calhoun P S., Stechuchak K M.., \& Strauss J, (2007). Interpersonal trauma, war zone exposure, and posttraumatic stress disorder among veterans with schizophrenia. Schizophrenia Research, 91, 210-216. 
14. Cary J., O'Donnell M L., \& Creamer M. (2006). Delayedonset PTSD: A prospective study of injury survivors. $J$. Affective Disorder. 90, 257-261.

15. Chandra, P. S., Satyanarayana, V.A., \& Carey , M. A. (2009). Women reporting intimate partner violence in India: Associations with PTSD and depressive symptoms, Archives of Women's Mental Health, 12, (4), 203-29.

16. Chemtob, C., Roitblat, H.L., Hamada, R.S., Carlson, J. G. \& Twentyman, C.T. (1988). A cognitive action theory of post-traumatic stress disorder. Journal of Anxiety Disorder, 2, 253-275.

17. Creamer, M., Burgess, P., \& Pattison, P. (1992). Reaction to trauma: A Cognitive processing model. Journal of Abnormal Psychology, 101, 452-459.

18. Dalglesh, T. \& Power, M.J. (1995). Theoretical approaches to posttraumatic stress disorder: The SPAARS model. Paper presented at the forth European Conference on traumatic stress, Paris. France, 7-11, MAY.

19. Davidson, J.R.T. (1997). Biological therapies for posttraumatic stress disorder an overview. Journal of Clinical Psychiatry, 58, 29-32.

20. Dunmore, E., Clark, D. M, \& Ehlers, A. (1999). Cognitive factors involved in the onset and maintenance of post-traumatic stress disorder (PTSD) after physical or sexual assault. Behaviour Research and Therapy, 37, 809 -30 .

21. Ehlers A., Clark D M., Hackmann A., McManus F., Fennell M., Herbert C, \& Mayou R (2003). A randomized controlled trial of cognitive therapy, a self-help booklet, and repeated assessments as early interventions for posttraumatic stress disorder. Archives of General Psychiatry, 60, 1024-1031

22. Eriksson, C. B., Vande Kemp ,H., Gorsuch, R., Hoke, S., \& Foya, D.W. (2001). Trauma exposure and PTSD symptoms in international relief and development personnel. Journal of Traumatic Stress, 14 (1)., 205-212.I

23. Foa, E.B. \& Kozak, M J, (1986). Emotional processing of fear: Exposure to corrective information. Psychological Bulletin, 99, 20-35.

24. Foa EB. (2000). Psychosocial treatment of posttraumatic stress disorder. Journal of Clinical Psychiatry. 61, (5).4348

25. Foa, E.B, Riggs, D.S., \& Gershuny, B.S. (1995). Arousal, numbing, and intrusion: symptom structure of PTSD following assault. American Journal of Psychiatry, 152, 116-20.

26. Frueh B C, Buckley TC, \& Cusack K J, (2004).Cognitivebehavioral treatment for PTSD among people with severe mental illness: a proposed treatment model. Journal of Psychiatric Practice, 10, 26-38.
27. Gidron Y, Gal R, Freedman S, Twiser I, Lauden A, Snir Y, Benjamin J. (2001). Translating research findings to PTSD prevention: results of a randomized pilot study. Journal of Trauma Stress, 14,773-780

28. Goodman, M., \& Weiss, D.(1998). Double trauma: a group therapy approach for Vietnam Veterans suffering from war and childhood trauma. International Journal of Group Psychotherapy. 48 (1). 39 - 54.

29. Goodman LA, Rosenberg SD, Mueser KT, \& Drake R E. (1997).Physical and sexual assault history in women with serious mental illness: prevalence, correlates, treatment, and future research directions. Schizophrenia Bulletin, 23, 685-696.

30. Halligan S L, Michael T, Clark D M, \& Ehlers A (2003). Posttraumatic stress disorder following assault: the role of cognitive processing, trauma memory, and appraisals. Journal of Consulting and Clinical Psychology, 71, 419431.

31. Harris M. (1998). Trauma Recovery and Empowerment: A Clinician's Guide for Working With Women in Groups. New York: The Free Press.

32. Horowitz, M., Wilner, M.,\& Alvarez, W. (1979). Impact of Event Scale: A measure of subjective stress. Psychosomatic Medicine, 41, 209-218.

33. Horowitz,M.J .(1973). Phase-oriented treatment of stress responses syndromes. American Journal of Psychotherapy,27, 506-515

34. Horowwitz, M.J. (1997). Stress Response Syndromes. PTSD, Grief and Adjustment Disorders. Jason Aronson, Northvale, N J.

35. Jacobson, E. (1938). Progressive Relaxation. Chicago:university of chicago Press.

36. Jonathan R., \& T. Davidson (2003). Effective Management Strategies for Posttraumatic Stress Disorder .FOCUS, 1, 239-243

37. Kessler, R.C., Borges, G., Walters, E.E (1999). Prevalence of and Risk Factors for Lifetime Suicide Attempts in the National Comorbidity Survey. Archives of General Psychiatry, 1999, 56, 617-626.

38. Kessler RC, Chiu WT, Demler O, \& Walters E E . (2005). Prevalence, severity, and co morbidity of 12-month DSM$I V$ disorders in the National comorbidity Survey Replication. Archives of General Psychiatry, 62, 617-627.

39. Kinzie, J.D. and Goetz, \& R.R.(1996). A century of controversy surrounding Post-traumatic stress-spectrum syndrome: the impact of DSM-III and DSM-IV. Journal of Praumatic Stress, 9, 159-79.

40. Kumar, S. M., Murhekar, \& Manoj V., Subramanian, (2007). .Prevalence of Posttraumatic Stress Disorder in a Coastal Fishing Village in Tamil Nadu, India, After the December 2004 Tsunami. American Journal of Public Health, 97 (1). , 99-101 
41. Mayou RA, Ehlers A, \& Hobbs M. (2000). Psychological debriefing for road traffic accident victims: three-year follow-up of a randomized controlled trial. British Journal of Psychiatry, 176, 589-593

42. Mc Farlane AC., Bookless C., \& Air T. (2001).Posttraumatic stress disorder in a general psychiatric inpatient population. Journal of Trauma Stress, 14,633-645.

43. Mueser K T., Bolton E., \& Carty P C., (2007). The trauma recovery group: a cognitive-behavioral program for PTSD in persons with severe mental illness. Community Mental Health Journal. Jan 19, 33-38.

44. Mueser K T., Goodman L A., \& Trumbetta S L., (1998).rauma and posttraumatic stress disorder in severe mental illness. Journal of Consult Clinical Psychology, 66, 493-499.

45. Mueser, K T., Rosenberg, S D., \& Jankowski, M K, (2004). A cognitive-behavioral treatment program for posttraumatic stress disorder in severe mental illness. American Journal of Psychiatric Rehabilitation, 7, 107146.

46. Murray J., Ehlers A., \& Mayou R A (2002). Dissociation and post-traumatic stress disorder: two prospective studies of road traffic accident survivors. British Journal of Psychiatry, 180,363-368

47. National Collaborating Centre for Mental Health: PostTraumatic Stress Disorder: The Management of PTSD in Adults and Children in Primary and Secondary Care. London, National Institute for Clinical Excellence, 2005

48. Rosenberg, S D., Mueser, K T.., \& Jankowski, M K.., (2004). Cognitive-behavioral treatment of posttraumatic stress disorder in severe mental illness: results of a pilot study. American Journal of Psychiatric Rehabilitaion, 7,171-186.

49. Shapiro, F. (1995). Eye movement desensitization and reprocessing: basic principles, protocols, and procedures, Guilford Press, New York.
50. Smith, MY., Redd, W H., DuHamel, K.,. Vickberg, S J., \& Ricketts, P. (1999). Validation of the PTSD ChecklistCivilian version in survivors of bone marrow transplantation. Journal of Traumatic stress; 12(3)., 485499.

51. Switzer, G E., Dew M A., \& Thompson K.., (1999). Posttraumatic stress disorder and service utilization among urban mental health center clients. Journal of Trauma Stress, 12, 25-39.

52. The Canadian Forces (2002). Supplement of the Statistics, Canada. Canadian Community Health Survey

53. Weathers, F., Huska J., \& Keane T. (1991). The PTSD checklist Military Version (PCL-M). Boston, Mass: National Center for PTSD.

54. Wilson, D \& Barglow, P. ( 2009 ). PTSD Has Unreliable Diagnostic Criteria. Psychiatric Times, 26 (7).

55. World Health Organization (1992). International statistical classification of diseases and related health problems, $10^{\text {th }}$ revision. WHO, Geneva.

\section{Authors:}

1. K S Sengar, Additional Professor, Department of Clinical Psychology, RINPAS, Kanke, Ranchi

2. Archana Singh, Psychiatric Social Worker, Department of PSW, RINPAS, Kanke, Ranchi, Jharkhand

\section{Address for Correspondence:}

Dr K S Sengar, Additional Professor, Department of Clinical Psychology, RINPAS, KANKE, Ranchi. Email: achyutsengar9@gamil.com 